\title{
Low prevalence of fluoroquinolone resistance among patients with tuberculosis in the Philippines: results of a national survey
}

To the Editor:

Antimicrobial resistance (AMR) represents a global threat to public health and security. Misuse of antibiotics is the leading cause of AMR worldwide. Infections that are typically easily treatable can become life-threatening or even deadly. Multidrug-resistant (MDR) tuberculosis (TB) (defined as resistance to at least rifampicin and isoniazid, the two most powerful first-line anti-TB drugs) and extensively drug-resistant (XDR)-TB (defined as MDR-TB with additional resistance to any fluoroquinolone and a second-line injectable (SLI) agent) are forms of $\mathrm{TB}$ that are complex to treat, require longer and more toxic regimens [1], and have considerably worse prognosis and outcome [2, 3]. Resistance to anti-TB drugs poses a major challenge to ending the global TB epidemic by 2030 [4]. To estimate the burden of drug-resistant $\mathrm{TB}$, and plan diagnostic and treatment services, surveillance of drug resistance among $\mathrm{TB}$ patients has been conducted worldwide since the 1990s [5].

The Philippines is considered by the World Health Organization (WHO) as a high-burden country for TB as well as for MDR-TB, with an estimated 22000 lives lost due to TB and 30000 new MDR-TB and rifampicin-resistant TB cases emerging in 2016 [6]. As in most high TB burden countries, the capacity in the Philippines for routine drug susceptibility testing (DST) for all TB patients continues to pose a challenge. In such settings, WHO recommends periodic epidemiological surveys to estimate the burden of drug resistance and monitor time trends [5].

The first national anti-TB drug resistance survey was conducted in the Philippines in 2004, and detected a prevalence of MDR-TB of 4.0\% (95\% CI 2.9-5.5\%) among new TB cases and 20.9\% (95\% CI 14.3-29.0\%) among previously treated cases [7]. In 2012, a second national anti-TB drug resistance survey was implemented according to WHO guidelines [8]. Ethical approval was granted by the Institutional Review Board of the Research Institute for Tropical Medicine (RITM) of the Philippines. The survey was of cluster-based, cross-sectional design, and enrolled 1377 new patients and 194 previously treated patients with a positive culture for Mycobacterium tuberculosis complex. The prevalence of MDR-TB among new cases decreased compared to 2004, at 2.0\% (95\% CI 1.4-2.7\%). In previously treated cases, the prevalence was similar at $21.4 \%$ (95\% CI $15.6-28.7 \%)$. The statistically significant decrease in MDR-TB prevalence among new TB cases between 2004 and 2012 ( $\mathrm{p}=0.001)$ reflects the effectiveness of TB control efforts implemented in the country over the past 15 years, including the high coverage of TB diagnostic and treatment services throughout the country, high treatment success rates and the availability of second-line therapy for patients with MDR-TB [6].

In order to investigate levels of resistance to fluoroquinolones and pyrazinamide, a random selection of 991 M. tuberculosis culture isolates from the 2012 survey was sequenced, corresponding to 901 new and 90 previously treated cases. Capillary sequencing was performed on the quinolone resistance determining regions of $\operatorname{gyr} A$ and $\operatorname{gyr} B$ to investigate fluoroquinolone resistance and $p n c A$ promoter region for pyrazinamide resistance using Sanger technology (Thermo Fisher Scientific, Waltham, MA, USA). Sequencing output data were analysed using Bioedit (Ibis Biosciences; Abbott Company, Carlsbad, CA, USA) and Sequencher version 4.9 DNA sequence analysis software (Gene Codes Corporation, Ann Arbor,

@ERSpublications

Prevalence of fluoroquinolone resistance among Filipino TB patients is lower than in other countries in the region http://ow.ly/7ywW30ix74W

Cite this article as: Lim DR, Dean AS, Taguinod-Santiago MR, et al. Low prevalence of fluoroquinolone resistance among patients with tuberculosis in the Philippines: results of a national survey. Eur Respir J 2018; 51: 1702571 [https://doi.org/10.1183/13993003.02571-2017]. 
MI, USA). The sequences were aligned to the M. tuberculosis $\mathrm{H} 37 \mathrm{Rv}$ reference strain (GenBank accession number AL123456.3) for nucleotide base changes, insertions and deletions, along with corresponding amino acid changes. Mutations in genes were interpreted using a recently developed standardised approach to determining the association of mutations with drug resistance [9]. Phenotypic DST was also performed on all culture isolates on liquid DST (MGIT960) for levofloxacin at $1.5 \mu \mathrm{g} \cdot \mathrm{mL}^{-1}$ and SLI agents at the following concentrations: $2.5 \mu \mathrm{g} \cdot \mathrm{mL}^{-1}$ for kanamycin and capreomycin, and $1.0 \mu \mathrm{g} \cdot \mathrm{mL}^{-1}$ for amikacin. All phenotypic and genotypic testing were performed by the National Tuberculosis Reference Laboratory (NTRL) of the RITM. The NTRL participates annually in first-line and second-line DST proficiency testing administered by its Supranational TB Reference Laboratory, the Research Institute of Tuberculosis in Tokyo, Japan, as well as biannually by the Integrated Quality Laboratory Services in Villeurbanne, France. Data were analysed using version 14.0 of Stata (StataCorp, College Station, TX, USA). Proportions of resistance and $95 \%$ confidence intervals were calculated by logistic regression, specifying robust standard errors to account for the cluster-based survey design.

Results of DST are shown in table 1. Prevalence of resistance to pyrazinamide, as determined by $p n c A$ sequencing, was low among new cases and rifampicin-susceptible cases. The majority of pyrazinamide-resistant cases were also rifampicin resistant, with a prevalence of pyrazinamide resistance of 7.2\% (95\% CI 1.1-13.4\%) among this group. A similar pattern was observed for SLI agents. Among rifampicin-resistant patients, the prevalence of resistance to any SLI agent was 5.9\% (95\% CI 1.6-10.1\%). These findings are encouraging, as rifampicin-resistant patients can be easily detected by rapid technologies such as GeneXpert (Cepheid, Sunnyvale, CA, USA) and then undergo further DST to a wider range of drugs.

Surprisingly, resistance to levofloxacin was very low. Only two resistant cases were detected, one of which was rifampicin susceptible and the other rifampicin resistant. As the latter was susceptible to SLI agents, no cases of XDR-TB were detected. There was good correlation with gene sequencing of gyrA and gyrB, with no resistance-conferring mutations detected. Levofloxacin is a component of the second-line anti-TB treatment regimen in the Philippines [10]. Although this national survey did not capture patients from the private sector, other surveys in Asia with a similar design have found alarmingly high levels of fluoroquinolone resistance even among new $\mathrm{TB}$ patients in the public system [11]. This may be a consequence of the unregulated use of fluoroquinolones for treatment of pneumonia and uncomplicated respiratory infections in these countries, including Pakistan and Bangladesh. Strict laws, such as the Philippine Pharmacy Law (Republic Act 10918), forbid the dispensing of antibiotics without a doctor's prescription [12]. Tighter regulations on the availability and use of antibiotics in the Philippines may be the main reason for the low levels of fluoroquinolone resistance in this survey, as compared to other countries in the region. An additional factor could be related to the price of an average treatment course of fluoroquinolones in the Philippines (approximately USD 29 for 7 days of levofloxacin and USD 28 for 7 days of moxifloxacin), which is considerably higher than in other Asian countries. These high prices may represent a barrier for most of the population to access these antibiotics unless covered by a treatment programme or insurance scheme.

This survey has some limitations. Firstly, the survey was designed with the primary objective of determining resistance to rifampicin and isoniazid, not to other drugs. Due to logistic reasons, only a subset of the culture isolates were sequenced. A larger sample size may improve the precision of the prevalence estimates of resistance. Secondly, the sensitivity of sequencing to detect resistance to pyrazinamide is lower than that of phenotypic testing $[13,14]$. Nevertheless, sequencing may be a valuable tool for the surveillance of drug resistance. It provides a faster turnaround time for results for a range of drugs when compared to conventional phenotypic testing [15].

TABLE 1 Prevalence $(95 \%$ CI) of resistance to anti-tuberculosis (TB) drugs in the Philippines

Resistance pattern

Rifampicin-resistant TB
Multidrug-resistant TB
Pyrazinamide
Fluoroquinolone
Any second-line injectable

Patient group

\begin{tabular}{lccc}
\hline New & Previously treated & Rifampicin susceptible & Rifampicin resistant \\
\hline $2.6 \%(1.8-3.3 \%)$ & $29.1 \%(20.0-38.2 \%)$ & & \\
$2.0 \%(1.4-2.7 \%)$ & $21.3 \%(15.5-28.7 \%)$ & & \\
$0.7 \%(0.2-1.2 \%)$ & $6.7 \%(1.3-2.0 \%)$ & $0.4 \%(0-0.9 \%)$ & $7.2 \%(1.1-13.4 \%)$ \\
$0.1 \%(0-0.4 \%)$ & $0.1 \%(0-4.2 \%)$ & $0 \%(0-0.4 \%)$ & $0 \%(0-4.6 \%)$ \\
$1.5 \%(0.9-2.2 \%)$ & $1.1 \%(0-2.7 \%)$ & $1.1 \%(0.5-1.6 \%)$ & $5.9 \%(1.6-10.1 \%)$
\end{tabular}

": includes kanamycin, capreomycin and amikacin. 
This survey has important implications for preventing the emergence and spread of drug-resistant $\mathrm{TB}$, as well as AMR in general. Enforcement of strict legislation throughout the health sector on the use of antibiotics is critical for protecting global public health.

Dodge R. Lim ${ }^{1,2}$, Anna S. Dean ${ }^{3}$, Mary Rosary Taguinod-Santiago ${ }^{4}$, Angeli Borbe-Reyes ${ }^{1}$, Andrea Maurizio Cabibbe $\oplus^{5}$, Matteo Zignol ${ }^{3}$, Ramon P. Basilio ${ }^{1,2}$, Anna Marie Celina Garfin ${ }^{4}$ and Maria Cecilia G. Ama ${ }^{1,2}$

${ }^{1}$ National Tuberculosis Reference Laboratory, Research Institute for Tropical Medicine, Muntinlupa City, Philippines.

${ }^{2}$ RITM TB Study Group, Research Institute for Tropical Medicine, Muntinlupa City, Philippines. ${ }^{3}$ World Health Organization, Geneva, Switzerland. ${ }^{4}$ National Tuberculosis Control Program, Dept of Health, Manila, Philippines. ${ }^{5}$ IRCCS San Raffaele Scientific Institute, Milan, Italy.

Correspondence: Dodge R. Lim, National Tuberculosis Reference Laboratory, Research Institute for Tropical Medicine, 9002 Research Dr., Filinvest Corporate City, Alabang, Muntinlupa City, Metro Manila 1781, Philippines. E-mail: dodge. lim@gmail.com

Received: Dec 132017 | Accepted after revision: Feb 062018

Conflict of interest: None declared.

Support statement: We thank the Bill and Melinda Gates Foundation, the Republic of the Philippines General Appropriations Act, and the Global Fund to Fight AIDS, Tuberculosis and Malaria for financially supporting this survey. Funding information for this article has been deposited with the Crossref Funder Registry.

This article has been revised according to the correction published in the May 2018 issue of the European Respiratory Journal.

\section{References}

1 Falzon D, Schünemann HJ, Harausz E, et al. World Health Organization treatment guidelines for drug-resistant tuberculosis, 2016 update. Eur Respir J 2017; 49: 1602308.

2 Falzon D, Mirzayev F, Wares F, et al. Multidrug-resistant tuberculosis around the world: what progress has been made? Eur Respir J 2015; 45: 150-160.

3 Ahmad Khan F, Salim MAH, du Cros P, et al. Effectiveness and safety of standardised shorter regimens for multidrug-resistant tuberculosis: individual patient data and aggregate data meta-analyses. Eur Respir J 2017; 50: 1700061.

$4 \quad$ Uplekar M, Weil D, Lonnroth K, et al. WHO's new End TB strategy. Lancet 2015; 385: 1799-1801.

5 Zignol M, Dean AS, Falzon D, et al. Twenty Years of Global Surveillance of Antituberculosis-Drug Resistance. N Engl J Med 2016; 375: 1081-1089.

6 World Health Organization. Global tuberculosis report 2017 (WHO/HTM/TB/2017.23). Geneva, World Health Organization, 2017.

7 Philippine Nationwide Tuberculosis Drug Resistance Survey Team. Nationwide drug resistance survey of tuberculosis in the Philippines. Int J Tuberc Lung Dis 2009; 13: 500-507.

8 World Health Organization. Guidelines for surveillance of drug resistance in tuberculosis. www.who.int/tb/ publications/2015/drs_guidelines/en/ Date last updated: June, 2015. Date last accessed: October 5, 2017.

9 Miotto P, Tessema B, Tagliani E, et al. A standardised method for interpreting the association between mutations and phenotypic drug resistance in Mycobacterium tuberculosis. Eur Respir J 2017; 49: 1701354.

10 Department of Health. National Tuberculosis Control Programme: Manual of Procedures. 5th Edn. www.doh.gov. ph/sites/default/files/publications/MOP_Final_a.pdf Date last updated: 2014. Date last accessed: June 18, 2017.

11 Zignol M, Dean AS, Alikhanova N, et al. Population-based resistance of Mycobacterium tuberculosis isolates to pyrazinamide and fluoroquinolones: results from a multicountry surveillance project. Lancet Infect Dis 2016; 16: $1185-1192$.

12 Food and Drug Administration. Antimicrobial Resistance. DOH-FDA Advisory No. 2012-017. www.fda.gov.ph/ attachments/article/38/DOH-FDA_Advisory_No_2012-017.pdf Date last updated: November 29, 2012. Date last accessed: October 20, 2017.

13 Whitfield MG, Warren RM, Streicher EM, et al. Mycobacterium tuberculosis pncA polymorphisms that do not confer pyrazinamide resistance at a breakpoint concentration of 100 micrograms per milliliter in MGIT. J Clin Microbiol 2015; 53: 3633-3635.

14 Willby M, Sikes RD, Malik S, et al. Correlation between GyrA substitutions and ofloxacin, levofloxacin, and moxifloxacin cross-resistance in Mycobacterium tuberculosis. Antimicrob Agents Chemother 2015; 59: 5427-5434.

15 Zignol M, Cabibbe AM, Dean AS, et al. Genetic sequencing for surveillance of drug resistance in tuberculosis in highly endemic countries: a multi-country population-based study. Lancet Infect Dis; In press. 